\title{
THE STUDY OF NON-DESTRUCTIVE MEASUREMENT APPLE'S FIRMNESS AND SOLUBLE SOLID CONTENT USING MULTISPECTRAL IMAGING
}

Muhua Liu ${ }^{1,2}$, Duan Wumao ${ }^{2}$, Huaiwei Lin $^{2}$

${ }^{1}$ Key Laboratory of Nondestructive Test in Nanchang University of Aviation of China Education Ministry, 330063,Nanchang, P.R.China 330045

2 College of Engineering, Jiangxi Agricultural University, 330045 Nanchang, P.R.China 330045

Corresponding author, Address: College of Engineering, Jiangxi Agricultural University, 330045 Nanchang, P.R.China 330045, Tel: +86-791-3813260, Fax: +86-797-3813260,

Email: suikelmh@sohu.com

Abstract: Firmness and soluble solid content (SSC) are two important quality attributes. This researches investigated the feasibility of using multi-spectral imaging to non-destructive measuring the apple's firmness and SSC. The spectral imaging in wavelength of $632 \mathrm{~nm} 、 650 \mathrm{~nm} 、 670 \mathrm{~nm} 、 780 \mathrm{~nm} 、 850 \mathrm{~nm}$ and $900 \mathrm{~nm}$ were captured. The Lorentzian distribution (LD), Gaussian distribution (GD) and Exponential distribution (ED) with three parameters were used to fit scattering profiles for all wavelengths. LD was found to be the best function for fitting gray distribution of the image. The multi-linear regression model using Lorentzian parameters for predicting apple firmness and soluble solids content were built using best single wavelength, double wavelengths, three wavelengths and four wavelengths. The best model with three wavelengths was able to predict apple soluble solid content with $\mathrm{r}=0.831$, $\mathrm{SEC}=0.55^{\circ}$ Brix and predict apple firmness with $\mathrm{r}=0.880, \mathrm{SEC}=0.52 \mathrm{~N}$ with four wavelengths. Experimental results show that the multi-spectral scattering imaging has high potential as a nondestructive and rapid method to assess fruit internal quality.

Keywords: Apple, Spectral imaging, Soluble solid content, Firmness, Multi-linear regression

Please use the following format when citing this chapter:

Liu, M., Wumao, D. and Lin, H., 2009, in IFIP International Federation for Information Processing, Volume 294, Computer and Computing Technologies in Agriculture II, Volume 2, eds. D. Li, Z. Chunjiang, (Boston: Springer), pp. 1077-1086. 


\section{INTRODUCTION}

Firmness and soluble solid content (SSC) are two important quality attributes used for assessing apple quality. On-line sensing using spectral imaging with several spectral bands can provide rapid method to assess fruits for their internal quality including firmness and SSC for human consumption, and thus, improve industry competitiveness and profitability.

Recently, optical techniques, especially near-infrared (NIR) spectroscopy, have been investigated as a nondestructive means for assessing quality attributes of fruits such as firmness and soluble solids content (SSC) (Lammertyn et al., 2000; Lu, 2000a). NIR spectroscopy determines the internal quality of fruit by measuring spectral reflectance/transmittance over the visible and NIR region. Although NIR spectroscopy has been successful for measuring SSC from whole fruit (Kawano et al., 1993; Lu, 2001; Slaughter, 1995).

McGlone et al. (1997) reported a moderately good correlation between scattering measurements and firmness, but with a high standard error. These reported studies have shown that light scattering is related to the condition of fruit. Diode lasers as a light source are easy to implement and low in costs, but they only provide light scattering information at one spectral band (or wavelength), which are insufficient for predicting fruit quality. Multiple wavelengths are needed in order to obtain more useful information about fruit firmness and SSC (Y Peng, 2006).

In this research, a spectral imaging technique was investigated for measuring light scattering profiles from apple fruit at selected wavelength $632 \mathrm{~nm}, 650 \mathrm{~nm}, 670 \mathrm{~nm}, 780 \mathrm{~nm}, 850 \mathrm{~nm}$ and $900 \mathrm{~nm}$. Computer algorithms were developed to quantify the spectral scattering images and relate them to fruit firmness and SSC.

\section{MATERIALS AND METHODS}

\subsection{Apple samples}

One hundred fresh Red Delicious (RD) apple samples were used for experiments. They were purchased from fruit market. The apples were stored in either controlled humidity environment $56 \%$ to $58 \%$ or room temperature from $24^{\circ} \mathrm{C}$ to $26^{\circ} \mathrm{C}$ for at least 20 hours prior to testing. Then, the apple samples were cleaned and marked before experiments were started. 


\subsection{Spectral imaging system}

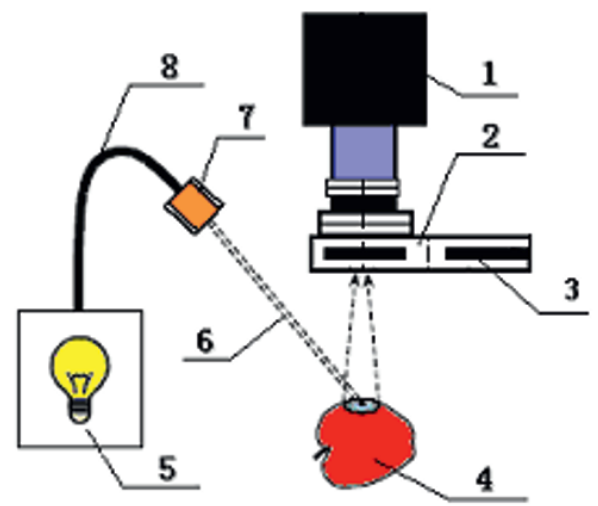

1. CCD Camera, 2.filter wheel setup, 3. filter, 4.fruit, 5.light source, 6.photon, 7.lens, 8.light fiber Fig. 1 Spectral Imaging System

Figure 1 shows a compact laboratory spectral imaging system. This system mainly consists of $250 \mathrm{~W}$ quartz tungsten light source, a high performance air-cooled CCD camera (Model GC-755P,HoneyWell Corp., Korea) with the pixel resolution of $0.1 \mathrm{~mm}$ per pixel, a rotating filter wheel containing six bandpass filters $(632,650,670,780,850$ and $900 \pm 10 \mathrm{~nm}$,) Matrox Meteor II/MC frame grabber (Matrox Corp., Canada)and computer with Intellicom image capture software. During image acquisition, each of the six filters was rotated in sequence to obtain six images. The exposure time was set at $1.2 \mathrm{~s}$ for the six filters. As the light beam hit the apple surface, most of the light penetrated into the fruit and scattered in different directions, which generated scattering images at the surface of the apple sample. The Spectral imaging system captured the scattering images of different wavelength through the filters from the apple surface over a $30 \mathrm{~mm}$ diameter area.

\subsection{Reference measurements}

\subsubsection{Firmness reference measurement}

Firmness was then measured from the same imaging location by using an $9 \mathrm{~mm}$ probe fruit sclerometer (Model GY-1 Top Instrument Corp., China) at a loading rate of $2.0 \mathrm{~mm} / \mathrm{s}$.Maximum forces recorded during $9 \mathrm{~mm}$ penetration were used as a reference measure of apple firmness. Firmness was tested for three times from the same imaging location then averaged as 
apple firmness value. All of apple samples were tested, they were divided into calibration set with 75 samples and validation set with 25 samples, Statistics of firmness data for the apple samples are shown in the table 1. As seen from the table 1, the range of reference measurement value in the calibration set covers the range of in the validation set, at the same time, the mean and standard derivation of the reference measurement in the calibration set are close to them in the validation set. Therefore, the distribution of samples is appropriate in calibration and validation sets.

Tab. 1 Reference measurement results of apple firmness (N)

\begin{tabular}{ccccc}
\hline Apple firmness & Mean & STD & Minimum & Maximum \\
\hline $\begin{array}{c}\text { Calibration set } \\
(\mathrm{n}=75)\end{array}$ & 8.91 & 0.92 & 7.0 & 11.1 \\
Validation set $(\mathrm{n}=25)$ & 8.65 & 1.20 & 7.6 & 10.5 \\
\hline
\end{tabular}

\subsubsection{SSC reference measurement}

SSC was measured from the same imaging location by using sugar refractometer (Model WZ-103 Zhongyou Optical Instrument Corp., China).SSC was tested for two times from the same imaging location then averaged as apple SSC value. All of apple samples were tested; they were divided into calibration set with 75 samples and validation set with 25 samples, Statistics of SSC data for the apple samples are shown in the table 2. As seen from the table 1, the range of reference measurement value in the calibration set covers the range of in the validation set, at the same time, the mean and standard derivation of the reference measurement in the calibration set are close to them in the validation set. Therefore, the distribution of samples is appropriate in calibration and validation sets.

Tab. 2 Reference measurement results of apple SSC ( ${ }^{\circ}$ Brix)

\begin{tabular}{ccccc}
\hline Apple sugar & Mean & STD & Minimum & Maximum \\
\hline $\begin{array}{c}\text { Calibration set } \\
(\mathrm{n}=75)\end{array}$ & 150 & 1.24 & 9.00 & 15.0 \\
Validation set $(\mathrm{n}=25)$ & 14.8 & 1.21 & 10.0 & 14.8 \\
\hline
\end{tabular}

\section{RESULTS AND DISCUSSION}

\subsection{Models of Scattering Profiles}

All of the apple samples were placed on the carry flat roof of spectral imaging system, and then each of the six filters was rotated in sequence to obtain six images. Six wavebands were 632nm, 650nm, 670nm, 780nm, 
$850 \mathrm{~nm}$ and $900 \mathrm{~nm}$ respectively. Six hundred scattering images were acquisition in all. Figure 2 show part of spectral scattering images.

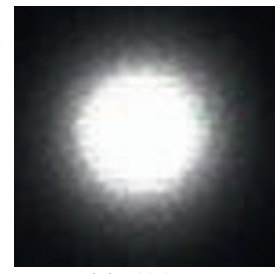

(a) $632 \mathrm{~nm}$

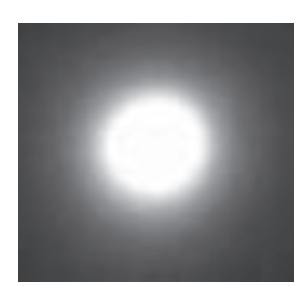

(c) $900 \mathrm{~nm}$

Fig. 2 spectral images o ${ }^{2}$ f apple $\quad$ Fig. 3 quantitative scattering image with pixels

Scattering images were circular and symmetric with respect to the light incident point, and their intensity decreased rapidly as the distance from the light incident point increased. Thus, each spectral scattering imaging could be reduced to a one-dimensional scattering profile through radial distance. In this study, the scattering image is divided into $\mathrm{N}$ circular bands of equaled distance or pixels, as shown in Fig3. The radial intensity of the scattering profiles was calculated by averaging all pixels within each circular, and every circular was achieved at intervals of eight pixels in turn. The following three mathematical models, i.e. Lorentzian distribution (LD), Gaussian distribution (GD) and Exponential distribution (ED) functions, were proposed comparatively to fit each scattering profile respectively. The LD, GD and ED functions are as follows Eqs.1-3,

$$
\begin{aligned}
& I_{i}=a_{1 i}+\frac{a_{2 i}}{1+\left(\frac{x}{a_{3 i}}\right)^{2}} \\
& I_{i}=a_{1 i}+a_{2 i} e^{-\frac{|x|}{a_{3 i}}} \\
& I_{i}=a_{1 i}+a_{2 i} e^{-0.5\left(\frac{x}{a_{3 i}}\right)^{2}}
\end{aligned}
$$

where: $\mathrm{I}$ is the light intensity in the CCD count; $\mathrm{x}$ is the scattering distance measured from the beam incident center, in $\mathrm{mm} ;{ }^{i}$ denote six different fitters, and $i=1,2, \ldots, 6 a_{1 i}, a_{2 i}$ and $a_{3 i}$ are asymptotic values, peak values, and scattering widths, corresponding to individual filters.

Figure 4 illustrates how the three distribution functions fit the scattering profiles at the six wavelengths for an apple fruit. When the distance (x) is small, the three distribution functions have a steep descending attribute; as $\mathrm{x}$ 
increase, they have a gentler descending attribute. The ED curve was sharp at the origin, and overall it did not fit the data well. The GD curve, on the other hand, under fit the section of the profile where greatest changes in the slope took place. LD had moderate slopes at the section adjacent to the peak and fitted the entire profile well. Among the three distributions studied. LD had the best curve-fitting results with the highest correlation and lowest stand error of estimate. The same data-fitting trend was found when the three distribution functions were applied to other samples.

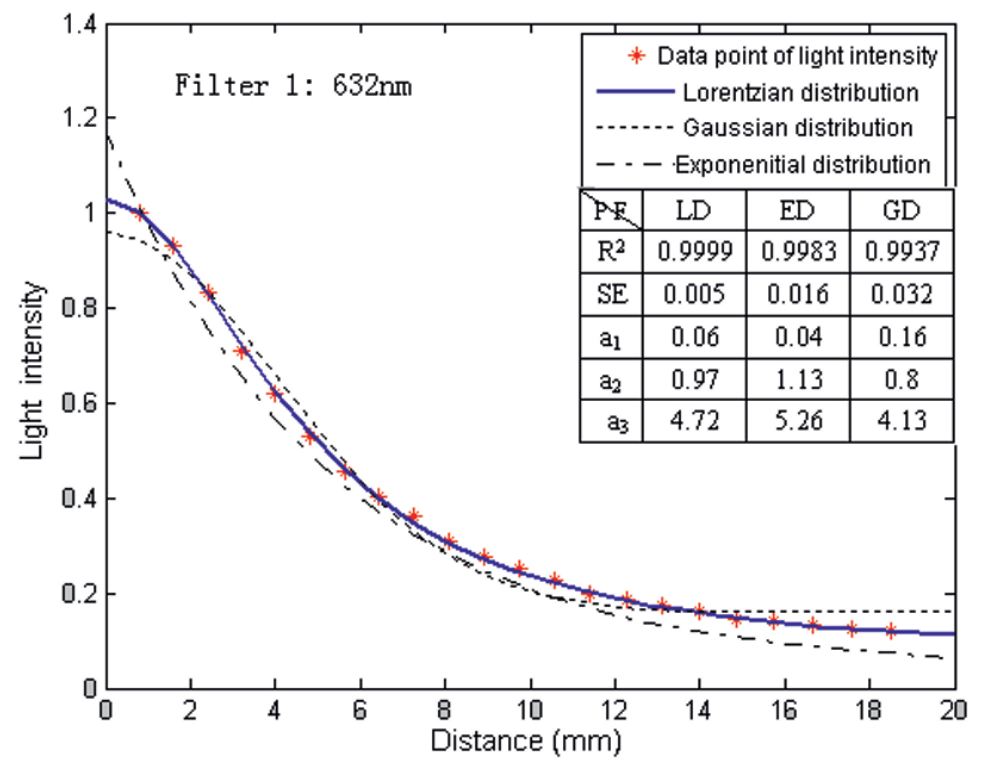

Fig. 4 Curve fittings of functions for an apple imaging scattering profiles in $632 \mathrm{~nm}$

\subsection{Predicting model building}

According to LD parameters and reference measurement results, the firmness and SSC prediction models were built by multi-linear regression (MLR) respectively. The prediction model consists of six partitions corresponding to six wavebands filters. Each partition has three LD parameters for an individual filter. The prediction model is described by the following equation:

$$
\hat{Y}=f_{0}+\sum_{i=1}^{N}\left(f_{1 i} a_{2 i}+f_{2 i} a_{2 i}+f_{3 i} a_{3 i}\right)
$$

where $\mathrm{Y}$ is the predictive results of model; $\mathrm{N}$ is the total number of filters; $\mathrm{i}=1,2,3,4,5,6 ; \mathrm{a}_{1 \mathrm{i}}, \mathrm{a}_{2 \mathrm{i}}$ and $\mathrm{a}_{3 \mathrm{i}}$ are asymptotic values, peak values, and scattering widths for $\mathrm{LD}$, corresponding to individual filters. $\mathrm{f}_{0}, \mathrm{f}_{1 \mathrm{i}}, \mathrm{f}_{2 \mathrm{i}}$ and $\mathrm{f}_{3 \mathrm{i}}(\mathrm{i}=1,2,3,4,5,6)$ are 19 regression coefficients. 
The prediction model calibrations and validations were performed. The procedures of establishing a firmness calibration model were as follows: (1) LD parameters $\left(a_{1 i}, a_{2 i}\right.$ and $\left.a_{3 i}\right)$ for Filter 1 through Filter 6 were calculated for each sample from the six spectral scattering images; (2) the 100 samples were divided into two groups randomly: 75 samples for calibration and the remaining 25 samples for validation; (3) A cross-validation method, in which one sample was left out each time, was applied to the calibration data set to remove extreme samples, as a result, about $8 \%$ of the total calibration samples were removed with 69 samples left for calibration; (4) The predictive models were built under six different wavebands according to Eq.4. The performance of the final MLR predictive models are evaluated in terms of standard error of calibration (SEC) and standard error of prediction (SEP) and the correlation coefficient (r).

\subsubsection{Model of apple firmness}

According to the Eq.4, the model of apple firmness was built with multilinear regression as follows,

$$
F=f_{0}+\sum_{i=1}^{N}\left(f_{1 i} a_{2 i}+f_{2 i} a_{2 i}+f_{3 i} a_{3 i}\right)
$$

where $\mathrm{F}$ is predictive results of firmness.

Multi-linear regression analysis indicated that when only one wavelength was used, the first wavelength $(680 \mathrm{~nm})$ gave best firmness predictions among the six wavelengths, with $\mathrm{r}$ equal to 0.698 and $\mathrm{SEP}=0.60 \mathrm{~N}$ for validation samples. Firmness predictions from the other five wavelengths were not as good as those from the first one, with $\mathrm{r}$ being between 0.423 and 0.686 . When two wavelengths were used, improved firmness predictions were observed: $\mathrm{r}=0.805$ and $\mathrm{SEP}=0.51 \mathrm{~N}$ for the validation samples. Firmness predictions continued to improve when three or four wavelengths were used, but the improvements were much smaller in terms of $r$ and SEP values. There results indicated that the best single wavelength was $632 \mathrm{~nm}$ with $\mathrm{r}=0.706$ and $\mathrm{SEC}=0.59$; the best double wavelengths combination were $650 \mathrm{~nm}$ and $900 \mathrm{~nm}$ with $\mathrm{r}=0.837$ and $\mathrm{SEC}=0.46$; the best three wavelengths combination were $632 \mathrm{~nm}, 650 \mathrm{~nm}$ and $850 \mathrm{~nm}$ with $\mathrm{r}=0.869$ and $\mathrm{SEC}=0.52$; the best four wavelengths combination were $632 \mathrm{~nm}, 650 \mathrm{~nm}, 670 \mathrm{~nm}$ and $900 \mathrm{~nm}$ with $\mathrm{r}=0.880$ and $\mathrm{SEC}=0.52$. More wavelength tend to improve prediction results, but the improvement is much smaller. When four wavelength combinations were used, the correlation coefficient was 0.880 for calibration and 0.869 for validation, and the SEC and SEP were $0.52 \mathrm{~N}$ and $0.53 \mathrm{~N}$, respectively. 
Through compared with best single wavelength, double wavelengths, three wavelengths and four wavelengths, the best four wavelengths combination has the best calibration and validation results for firmness prediction of apple fruit by a multi-linear regression, then the firmness prediction model was developed.

\subsection{SSC Prediction Model}

According to the Eq.4, the model of apple SSC was built with multi-linear regression as follows,

$$
S=f_{0}+\sum_{i=1}^{N}\left(f_{1 i} a_{2 i}+f_{2 i} a_{2 i}+f_{3 i} a_{3 i}\right)
$$

where $\mathrm{S}$ is predictive results of SSC.

Multi-linear regression analysis indicated that when only one wavelength was used, the first wavelength $(680 \mathrm{~nm})$ gave best SSC predictions among the six wavelengths, with $r$ equal to 0.610 and $\mathrm{SEP}=0.61 \mathrm{~N}$ for validation samples. SSC predictions from the other five wavelengths were not as good as those from the first one, with $\mathrm{r}$ being between 0.336 and 0.564 . When two wavelengths were used, improved SSC predictions were observed: $r=0.761$ and $\mathrm{SEP}=0.64 \mathrm{~N}$ for the validation samples. Firmness predictions continued to improve when three or four wavelengths were used, but the improvements were much smaller in terms of $r$ and SEP values. There results indicated that the best single wavelength was $632 \mathrm{~nm}$ with $\mathrm{r}=0.622$ and $\mathrm{SEC}=0.58$, best double wavelengths combination were $632 \mathrm{~nm}$ and $650 \mathrm{~nm}$ with $\mathrm{r}=0.776$ and SEC $=0.62$; best three wavelengths combination were $632 \mathrm{~nm}, 650 \mathrm{~nm}$ and $780 \mathrm{~nm}$ with $\mathrm{r}=0.831$ and $\mathrm{SEC}=0.55$; best four wavelengths combination were $650 \mathrm{~nm}, 670 \mathrm{~nm}, 780 \mathrm{~nm}$ and $850 \mathrm{~nm}$ with $\mathrm{r}=0.813$ and $\mathrm{SEC}=0.67$. More wavelengths tend to improve prediction results, but the improvement is much smaller. When four wavelength combinations were used, the correlation coefficient was 0.813 for calibration and 0.805 for validation, and the SEC and SEP were $0.67 \mathrm{~N}$ and $0.68 \mathrm{~N}$, respectively. However the three wavelength combination with $\mathrm{r}=0.831$ for calibration and $\mathrm{r}=0.819$ for validation, and the SEC and SEP were $0.55 \mathrm{~N}$ and $0.55 \mathrm{~N}$, respectively.

Through compared with best single wavelength, double wavelengths, three wavelengths and four wavelengths, the best three wavelengths combination has the best calibration and validation results for SSC prediction of apple fruit by a multi-linear regression, then the SSC prediction model was developed. 


\section{CONCLUSIONS}

Spectral scattering images at six selected wavelengths were useful for predicting apple fruit firmness and SSC. Comparing three mathematical functions i.e. $\mathrm{LD}, \mathrm{ED}$, and $\mathrm{GD}, \mathrm{LD}$ was the best for fitting the scattering profiles for Red Delicious apples acquired by a spectral imaging system.

Parameters of the LD were linearly related to fruit firmness and SSC. Multi-linear prediction model was established between LD parameters and fruit firmness and SSC. This research demonstrated that the best model with three wavelengths was able to predict apple SSC with $\mathrm{r}=0.831$, SEC $=0.55$ and four wavelengths was able to predict apple firmness with $\mathrm{r}=0.880$, $\mathrm{SEC}=0.52$. Spectral scattering technique is a promising technique for nondestructive sensing of apple fruit firmness and SSC. Further research is being conducted to improve the spectral imaging system for real time acquisition of scattering images from apple fruit and develop more effective and efficient algorithms to classify fruit into many different grades based on their firmness and SSC.

\section{ACKNOWLEDGEMENTS}

This work has been financially supported by the National Natural Science Foundation of China (No. 30460059) and The Science Foundation of Key Laboratory of Nondestructive Test in Nanchang University of Aviation of China Education Ministry for assistance.

\section{REFERENCES}

D. C. Slaughter, 1995, Nondestructive Determination Of Internal Quality In Peaches And Nectarines. Transactions Of The Asae, 38(2): 617-623.

J. Lammertyn, B. Nicolai, K. Ooms, V. De Smedt, J. De Baerdemaeker, 1998, Nondestructive Measurement Of Acidity, Soluble Solids, And Firmness Of Jonagold Apples Using NirSpectroscopy. Transactions Of The Asae 41(4): 1089-1094.

R. Lu, 2001, Predicting Firmness And Sugar Content Of Sweet Cherries Using Near-Infrared Diffuse Reflectance Spectroscopy. Transactions Of The Asae, 44(5): 1265-1271.

R. Lu, D.E. Guyer, R.M. Beaudry, 2000, Determination Of Firmness And Sugar Content Of Apples Using Near-Infrared Diffuse Reflectance. J. Texture Stud., 31: 615-630.

S. Kawano, H. Watanabe, M. Iwamoto, 1992, Determination Of Sugar Content In Intact Peaches By Near Infrared Spectroscopy With Fiber Optics In Interactance Mode. J. Japan. Hort. Sci. 61(2): 445-451.

V.A. Mcglone, H. Abe, S. Kawano, 1997, Kiwifruit Firmness By Near Infrared Light Scattering. J. Nir Spectros. 5: 83-89. 
Y. Peng, R. Lu, 2006, An Lctf-Based Multispectral Imaging System For Estimation Of Apple Fruit Firmness: Part I. Acquisition And Characterization Of Scattering Images. Transactions Of The Asae. 49(1): 259-267.

Y Peng, R Lu, 2006, An Lctf-Based Multispectral Imaging System For Estimation Of Apple Fruit Firmness: Part Ii. Selection Of Optimal Wavelengths And Development Of Prediction Models. Transactions Of The Asae. 49(1): 269-275. 